\title{
Extensions of Ip-norm optimum filters for image recognition
}

Nasser Towghi, Bahram Javidi

Nasser Towghi, Bahram Javidi, "Extensions of Ip-norm optimum filters for image recognition," Proc. SPIE 10296, 1999 Euro-American Workshop Optoelectronic Information Processing: A Critical Review, 102960B (2 June 1999); doi: 10.1117/12.365908

SPIE Event: Euro-American Workshop on Optoelectronic Information Processing, 1999, Colmar, France 


\title{
Extensions of $l_{p}$-norm optimum filters for image recognition
}

\author{
Nasser Towghi and Bahram Javidi \\ Department of Electrical and System Engineering \\ University of Connecticut, U-157, CT 06269-3357
}

\begin{abstract}
A family of linear and nonlinear processors (filters) for image recognition, which are extensions of the previously developed filters called $l_{p}$-norm optimum filters, are presented. These filters are $l_{p}$-norm optimal in terms of tolerance to input noise and discrimination capabilities. The $l_{p}$-norm is the generalization of the usual mean squared $\left(l_{2}\right)$ norm, obtained by replacing the exponent 2 by any positive constant $p$ (usually $p \geq 1$ ). These processors are developed by minimizing the $l_{p}$-norm of the filter output due to the input scene and the output due to input noise. The minimization is carried out by constraining a function of the filter output to attain a fixed peak value when the input is the target to be detected.
\end{abstract}

The use of $l_{p}$-norm to measure the size of the filter output due to noise gives a greater freedom in adjusting the noise robustness and discrimination capabilities. The flexibility in allowing more general type of constraints allows for experimenting and may lead to designing of filters to obtain better performance by selecting an appropriate filter constraint equation to match the metric used

1999 Euro-American Workshop Optoelectronic Information Processing: A Critical Review, edited by Philippe Refreglier, Bahram Javidi, Proc. of SPIE Vol. 10296 (Vol. CR74), 102960B (C) (1999) 2017 SPIE · CCC code: 0277-786X/17/\$18 - doi: 10.1117/12.365908 
to measure the performance of the filter.

we give an unified theoretical basis for developing these filters. This family of filters include some of the existing linear and nonlinear filters.

Key words: $l_{p}$-norm filters, nonlinear filters, pattern recognition, signal detection.

\section{INTRODUCTION}

In this paper we review and extend a family of filters developed for image recognition. A family of filters termed $l_{p}$-norm optimum filters were designed based on minimizing the output due to the input signal and the output due to the input noise. ${ }^{1}$ To measure the size of the output, $l_{p}$-norm metric was used. Theoretical development of these filters is given in detail in reference [1]. In this paper paper we extend the results obtained in [1], by constraining the filter output or a nonlinear function of the filter output to attain a fixed peak value when the input is the target to be detected. This is explained in section 3 .

Numerous types of filters have been developed for image and pattern recognition, for instance the matched filter ${ }^{2}$ and its variations (see e.g., references [3-9]). Matched filter maximizes the signal to noise ratio in the presence of stationary additive noise, but it it is shown to have low discrimination capabilities. ${ }^{5}$ On the other hand, variations of these filters have shown to be discriminant with 
1999 Euro-American Workshop on Optoelectronic Information Processing / 243

good correlation performance. ${ }^{4,8,10,11}$ Various other filter based on different design criteria have been proposed to optimize some criteria or for compromise between different criteria. ${ }^{12-16}$

Recently we developed a family of filters for image recognition, which were based on minimizing output due to the input signal and the output due to the input noise. ${ }^{1}$ We used the $l_{p}$-norm to measure the output. By choosing to minimize both the $l_{p}$-norm of the output due to noise and the output due to the input scene; or either one of the two; and using different values of $p$, we generated a family of filters indexed by parameters $q=\frac{p}{p-1}$ and $\sigma$, where $q$ controls the discrimination and robustness of the filter and $\sigma$ is the standard deviation of the additive noise.

In this paper we extend the development of $l_{p}$-norm optimum filters, by allowing more general type of constraint on the filter output when the input is the target. This allows us to generate a family of filters. The family is indexed by parameters $q, \sigma$, and $f$ where $q=\frac{p}{p-1}$ is positive scalar which controls the discrimination and robustness of the filter, $\sigma$ is the standard deviation of the additive noise, and $f$ is a functional defining the constraint equation when the input to the processor is the target itself (see section 3). A special type of constraint function $f$ gives us two general families of filters, one indexed by parameters $(\sigma, q, b, c)$ and the second is indexed by parameters $(u, d)$. These two family of filters will include many of the long existing, as well as, some of the more recently proposed filters. Thus, the results of this paper provide 
the mathematical justification of some of the novel filtering ideas which have recently been proposed.

In subsection (3.3) we list some of the more well known filters, which can be obtained by the family of filters developed in this paper, by choosing the appropriate parameters in our equations describing these filters. In particular, the class of filters developed here includes and extend the $k$ th law nonlinear filters $^{7}$, as well as, the more general form of the $k$ th law nonlinear filters, known as dual nonlinear correlators. ${ }^{17}$ The family developed in this paper also includes the family of the $l_{p}$-norm filters developed previously by the authors. ${ }^{1}$ The previously developed $l_{p}$-norm filters include the classical matched filter, the phase only filter, ${ }^{4}$ and the adaptive image discriminating and noise robust nonlinear processor of Refegier, Laude and Javidi. ${ }^{11}$

The new idea in this paper is to allow more general type of constraint equation with respect to which, the minimization of expressions controlling the discrimination capabilities and noise robustness are carried out. Since the function $f$ describing the constraint equation can be arbitrary and general (with some restriction as explained in section 3 ), one obtains a great deal of flexibility, and by judicious choice of $f$, perhaps one can obtain optimum results by relating the functional $f$ to the metric used to measure the performance of the filters. As mentioned a particular choice of $f$ leads to $k$-law nonlinear filters, which have been shown to be more discriminant than the usual matched filter.

Paper is organized as follows. In section 2 we briefly state and review the 
1999 Euro-American Workshop on Optoelectronic Information Processing / 245

minimization problem which is the basis of $l_{p}$-norm filters. ${ }^{1}$ Since the theoretical development of these filters is along the lines of filters developed which appears in reference [1], we simply state the final results. In section 3 we extend the theoretical developments of the $l_{p}$-norm filters by imposing a general constraint on the output of the filter when the input is the target to be detected. In subsection 3.1 we consider very general type of constraints given by a function $f$ and obtain a family of filters denoted by $H_{(q, f)}^{\sigma}$. In subsection 3.2 we consider special types of constraining functions $f$. This gives us a related family of filters denoted by $H_{(q, b, c)}^{\sigma}, H_{(q, b, c)}^{0}, H_{(q, b, c)}$ and $H_{(u, d)}$. In subsection 3.3 , we list some of the more popular filters and other existing filters which are special cases of the classes of filters developed in subsection 3.2. In section 4 some simulation results are presented conclusions are presented in section 5 .

\section{ANALYSIS}

In this section we review the development of the $l_{p}$-norm filters, ${ }^{1}$ and collect the necessary background materials. We refer the reader to [1] for a detailed analysis.

Let $r(j)$ denote a target to be detected, and $n(j)$ the additive noise, which we assume to be zero mean and white stationary. Then the input to the system (filter) is

$$
s(j)=r(j)+n(j)
$$


Let $S(k), R(k)$, and $N(k)$ denote the Fourier transforms of $s(j), r(j)$, and $n(j)$, respectively.

Let $h$ denote the impulse response function of the system and $H$ denote the Fourier transform of $h$. The filter $h(j)$ is designed such that, the filter output due to the target $r$ is

$$
\sum_{j=0}^{J-1} h(j)^{*} r(j)=C(0)
$$

where $C=C(0)$ is a positive constant. To achieve both robustness and discrimination capabilities, the filter $h(j)$ is designed to minimize a weighted sum of the $p$ th power of $l_{p}$-norms mean of the output due to noise $n$, and the $p$ th power of $l_{p}$-norm of the output due to input signal $s$. That is, $h$ is chosen to minimize,

$$
a \sum_{j=0}^{J-1} E\left|\sum_{l=0}^{J-1} h(j-l) n(l)\right|^{p}+b \sum_{j=0}^{J-1}\left|\sum_{l} h(j-l) s(l)\right|^{p}
$$

under the constraint of equation (2). The weights $a$ and $b$ are suitably chosen positive quantities. If the emphasis is on robustness, $b$ is the larger of the two. If the emphasis is on discrimination, $a$ should be the dominant quantity. We only consider the case, $(a=b=1),(a=1, b=0)$ and $(a=0, b=1)$.

The minimization problem given by equation (3) for the case $1<p \leq 2$, can be stated in Fourier domain ${ }^{1}$ : Minimize

$$
\sum_{j=0}^{J-1}|H(j)|^{q}\left(\hat{\sigma}_{q}+|S(j)|^{q}\right), \quad \text { subject to } \quad \sum_{j=0}^{J-1} H(j)^{*} R(j)=J C(0)
$$


1999 Euro-American Workshop on Optoelectronic Information Processing / 247

where $q=\frac{p}{p-1}$ and $\hat{\sigma}_{q}=E|N(j)|^{q}$.

\subsection{General Nonlinear filters based on optimization using $l_{p}$ norms}

The solution of the minimization problem of equation (4) is a constant multiple of $^{1}$

$$
H_{q}^{\sigma}(j)=\left[\frac{|R(j)|}{\hat{\sigma}_{q}+|S(j)|^{q}}\right]^{\frac{1}{q-1}} \exp \left(i \Phi_{R(j)}\right)
$$

where $\hat{\sigma}_{q}=E|N(j)|^{q}$ and $\Phi_{R(j)}$ is the argument (phase) of the complex quantity $R(j)$, that is, $R(j)=|R(j)| \exp \left(i \Phi_{R(j)}\right)$.

The case $p=1$ requires a different approach. We refer the reader to [1]. However, if we settle on using the lower bound estimate, $E|N(j)|^{q} \geq[\sigma \sqrt{J}]^{q}$ which holds for $q \geq 2$, we obtain a crude approximation of a filter equation for the case $q=\infty$ or $p=1$, given below, ${ }^{1}$

$$
H_{\infty}^{\sigma}(j)=\left[\frac{1}{\max \{\sqrt{J} \sigma,|S(j)|\}}\right] \exp \left(i \Phi_{R(j)}\right)
$$

We should point out that equation (5) requires the values of $E|N(j)|^{q}$. With few exception this quantity may be difficult to compute. The reader can find some lower bound and upper bound estimates for various types of noise processes in reference [1].

\subsection{Sub family of linear and nonlinear filters based on minimiz-} ing the $l_{p}$-norm

If we only minimize the filter output due to the input scene $s$, subject to equation 
(2), we obtain the following family of filters, which we denote by $H_{q}^{0}$,

$$
H_{q}^{0}(j)=|R(j)|^{\frac{1}{q-1}}|S(j)|^{\frac{1}{1-q}-1} \exp \left(i \Phi_{R(j)}\right)
$$

where $q \geq 2$, and

$$
H_{\infty}^{0}(j)=|S(j)|^{-1} \exp \left(i \Phi_{R(j)}\right)
$$

If we only minimize the output due to the additive noise, we obtain a filter denoted by $H_{q}$, where $q \geq 2$,

$$
H_{q}(j)=|R(j)|^{\frac{1}{g-1}} \exp \left(i \Phi_{R(j)}\right)
$$

and

$$
H_{\infty}(j)=\exp \left(i \Phi_{R(j)}\right)
$$

\section{EXTENSIONS OF THE $l_{p}$-NORM OPTIMUM FILTERS}

\subsection{General case}

We now extend the development of the $l_{p}$-norm optimum filters by allowing more general type of constraint on the output of the filters when the input is the target itself. More precisely, we replace the constraint given by Eq. (2), by the following more general type of constraint:

$$
f(H, R)=\frac{1}{J} \sum_{j=0}^{N-1} Q_{j}[|H(j)|] P_{j}[|R(j)|] \exp \left[i\left(\Phi_{R(j)}-\Phi_{H(j)}\right)\right]=C,
$$


where $H(j)$ is the filter transfer function and $R(j)$ is the Fourier transform of the target. $Q_{j}$ and $P_{j}$ are arbitrary real valued function of one variable selected at the discretion of the designer. Thus in Eq. (2), $Q_{j}[|H(j)|]=|H(j)|$ and $P_{j}[|R(j)|]=|R(j)|$. If we impose that, the functions $Q_{j}(\cdot)$ be differentiable, one to one (a function $g$ is one to one provided that, $g(x)=g(y)$ implies $x=y$ ), and the functions $G_{j}(t)=\frac{t^{q-1}}{Q_{j}^{\prime}(t)}$ have well defined inverses, then the minimization of Eq. (4) under the constraint of Eq. (11) can be transfered to Fourier domain. The details of transference of the minimization problem from spatial domain to Fourier domain and the solution of the minimization problem in Fourier domain with constraint prescribed by Eq. (11) are similar to the ones described in appendix A of reference [1]. As in [1], it can be shown that the solution of the minimization problem given by Eq. (4) but with the constraint given by Eq. (11), is a constant multiple of

$$
H_{(q, f)}^{\sigma}(j)=\left[G_{j}^{-1}\left(\frac{P_{j}(|R(j)|)}{\hat{\sigma}_{q}+|S(j)|^{q}}\right)\right] \exp \left(i \Phi_{R(j)}\right)
$$

where $G_{j}^{-1}(t)$ is the inverse of

$$
G_{j}(t)=\frac{t^{q-1}}{Q_{j}^{\prime}(t)}, \quad t>0
$$

If we minimize the filter output that is only due to the input scene $s$, subject to constraint of Eq. (11), we obtain the following family of filters, which we denote by $H_{(q, f)}^{0}$,

$$
H_{(q, f)}^{0}(j)=\left[G_{j}^{-1}\left(P_{j}[|R(j)|]|S(j)|^{-q}\right)\right] \exp \left(i \Phi_{R(j)}\right)
$$


where $q \geq 2$.

If we only minimize the output that is due to the additive noise, we obtain a filter denoted by $H_{(q, f)}$, where $q \geq 2$,

$$
H_{(q, f)}(j)=\left[G_{j}^{-1}\left(P_{j}[|R(j)|]|S(j)|^{-q}\right)\right] \exp \left(i \Phi_{R(j)}\right)
$$

\subsection{Special cases}

We now consider special types of the functions $Q_{j}$ and $P_{j}$ for the constraint equation. Consider the special case of constraint equation given by

$$
f(H, R)=\sum_{j=0}^{J-1}|H(j)|^{b_{j}}|R(j)|^{c_{j}} \exp \left[i\left(\Phi_{R(j)}-\Phi_{H(j)}\right)\right]=J C,
$$

where $b_{j}$ and $c_{j}$ are arbitrary constants with $b_{j} \neq 0$. With this constraint we obtain the family indexed by $q, b=\left(b_{0}, \ldots, b_{j-1}\right), c=\left(c_{0}, \ldots, c_{J-1}\right)$, and $\sigma$. In this case the filter which we obtain by minimizing Eq. (4) takes the following form,

$$
H_{(q, b, c)}^{\sigma}(j)=\left[\frac{|R(j)|^{c_{j}}}{\hat{\sigma}_{q}+|S(j)|^{q}}\right]^{\frac{1}{q-b_{j}}} \exp \left(i \Phi_{R(j)}\right)
$$

If we only minimize the filter output that is due to the input scene $s$, subject to constraint of Eq. (11), we obtain the following family of filters,

$$
H_{(q, b, c)}^{0}(j)=|R(j)|^{\frac{c_{j}}{q-b_{j}}}|S(j)|^{\frac{-q}{q-b_{j}}} \exp \left(i \Phi_{R(j)}\right)
$$

where $q \geq 2$. 
If we only minimize the output that is due to the additive noise, we obtain a filter denoted by $H_{(q, b, c)}$, where $q \geq 2$,

$$
H_{(q, b, c)}(j)=|R(j)|^{\frac{c_{j}}{q-b_{j}}} \exp \left(i \Phi_{R(j)}\right)
$$

The forms of equations of filters given by Eq. (18) and Eq. (19) suggest that by a judicious choice of $q, b=\left(b_{0}, \ldots, b_{J-1}\right)$, and $c=\left(c_{0}, \ldots, c_{J-1}\right)$, and by either minimizing the output that is due to the input noise or by minimizing the output that is due to the input scene, we can obtain any filter of the form,

$$
H_{(u, d)}(j)=|R(j)|^{d_{j}}|S(j)|^{u_{j}} \exp \left(i \Phi_{R(j)}\right)
$$

This type of filter had previously been proposed by Kotynski and ChalasinskaMacukow. ${ }^{17}$

\subsection{Summary and comparison with the popular filters}

In reference [1] we had derived class of linear and nonlinear filters defined by equations 5 thorough $10,(14),(15)$, and by equations (17) through (20).

(i) $H_{(q, b, c)}^{\sigma}$ family, given by equations (5), (6), and (17). Here $2 \leq q \leq \infty$.

The filters defined by these three equations are nonlinear, and developed to be both robust to noise and discriminant against false objects.

(ii) $H_{(q, b, c)}^{0}$ family, given by equations (7), (8), and (18). Here $2 \leq q \leq \infty$.

The filters defined by these three equations are nonlinear, and were derived to optimize their discrimination capabilities.

(iii) $H_{(q, b, c)}$ family, given by equations (9), (10), and (19). Here $2 \leq q \leq \infty$. 
The filters defined by these three equations are linear, and were derived to be robust to noise.

(iv) $H_{(u, d)}$ family of filters defined by Eq. (2).

This family is obtained by judicious choices of indices $q,, b$, and $c$ of the filters given by Eqs. (17) and (18).

Recall that these filters are obtained by minimizing the the output energy due to noise using $l_{p}$ norm as the metric, where $p=q /(q-1)$. Since $l_{p}$-norm is decreasing function of $p$, that is for $p_{1}>p_{2}$

$$
\|c\|_{p_{1}} \leq\|c\|_{p_{2}}
$$

we expect that filter becomes more discriminant as $q$ tends to infinity. This is also confirmed by computer simulations (see section 3 ).

We also note that, the aforementioned families of filters generalize many exiting filters. For instance $H_{2}^{\sigma}$ given by equation (11) is the filter derived in reference [8]. Let us recall that, the output of $k$ th law Fourier plane nonlinear filter at the Fourier domain

The aforementioned families of filters generalize many existing filters. For instance $H_{2,0}^{\sigma}$ given by equation (5) is the filter derived in reference [11]. Let us recall that, the output of $k$ th law Fourier plane nonlinear filter at the Fourier domain is given by 10

$$
C(j)=|R(j)|^{k}|S(j)|^{k} \exp \left(i\left(\Phi_{R(j)}-\Phi_{S(j)}\right)\right)
$$


where $0 \leq k \leq 1$. The output is obtained by taking the inverse Fourier transform of $C(j)$. Let

$$
H_{k}(j)=|R(j)|^{k}|S(j)|^{k-1} \exp \left(i \Phi_{R(j)}\right)
$$

then the output of the $k$ th law Fourier plane nonlinear filter is inverse Fourier transform of $C(j)$ where

$$
C(j)=H_{k}(j)^{*} S(j)
$$

Equations (7), (8), (20), and (22) show the similarity of the $k$ th law nonlinear filters with $H_{q}^{0}$ filters of Eq. (7) and $H_{(u, d)}$ filters of Eq. (22). In fact, $H_{\infty}^{0}$ filter is precisely the $k$ th law nonlinear filter for $k=0$, and by letting $d_{j}=k$ and $u_{j}=k-1$ in Eq. (22), one obtains the $k$ th law nonlinear filters.

In the table below we list some of the more popular filters which can be obtained by using different values for $\sigma, q, u_{j}$ and $d_{j}$.

Table 1. Popular filters obtained from $H_{q}^{\sigma}$ and $H_{(u, d)}$ family of filters.

\begin{tabular}{|l|l|l|}
\hline Popular filters & Family & Parameters \\
\hline$k$-th law nonlinear filters & $H_{(u, d)}$ & $u_{j}=k-1, d_{j}=k$ \\
\hline Phase only matched filter & $H_{(u, d)}$ & $u_{j}=0, d_{j}=0$ \\
\hline Binary JTC & $H_{(u, d)}$ & $u_{j}=-1, d_{j}=0$ \\
\hline Matched filter & $H_{(u, d)}$ & $u_{j}=1, d_{j}=0$ \\
\hline Dual nonlinear correlator & $H_{(u, d)}$ & $u_{j}=L-1, d_{j}=M$ \\
\hline Nonlinear JTC of [11] & $H_{q}^{\sigma}$ & $q=2$ \\
\hline Absolute mean value filter & $H_{q}^{\sigma}$ & $q=\infty$ \\
\hline
\end{tabular}


254 / Critical Reviews Vol. CR74

\section{COMPUTER SIMULATIONS}

To test the performances of the filters designed in section 2 , we have performed some computer simulations. In our simulations our target is a jet airplane. The size of the target is $107 \times 70$ pixels as shown in Fig. 1. The target is placed in scene containing color background noise background and additive white Gaussian noise (AWGN) as shown in Fig. 2. The additive noise is stationary with mean, $\mu=0$ and standard deviation, $\sigma=.5$. The bandwidth of the background noise is $50 \times 50$ pixels, and its mean at each pixel as .2 and its standard deviation at each pixel is .2 . The size of the scene is $256 \times 256$ pixels.

Figures 3 and 4 show the output of the $H_{q}^{\sigma}$ family of filters given by Eqs. (5). Figure 3 is the output of the filter when $q=2$, figure 4 is the output of the filter when $q=10$. The set of filters whose output is given by figures 2(a) and 2(c) were designed to optimize both the noise robustness and discrimination capabilities. We see that the correlation peaks are sharper for larger values of

$q$.

Figures 5 through 7 show the output of the $H_{q}^{0}$ family of filters given by Eqs. (7) and (8). Figure 5 is the output of the filter when $q=2$, figure 6 is the output of the filter when $q=10$, and figure 7 is the output of the filter when $q=\infty$, given by Eq. (8). The set of filters whose output is given by figures 5 through 7 were designed to optimize both the noise robustness and discrimination capabilities. Once again we see that the correlation peaks are sharper for larger values of $q$. Since in the derivation of $H_{q}^{0}$ filters only the $l_{p}$ 
1999 Euro-American Workshop on Optoelectronic Information Processing / 255

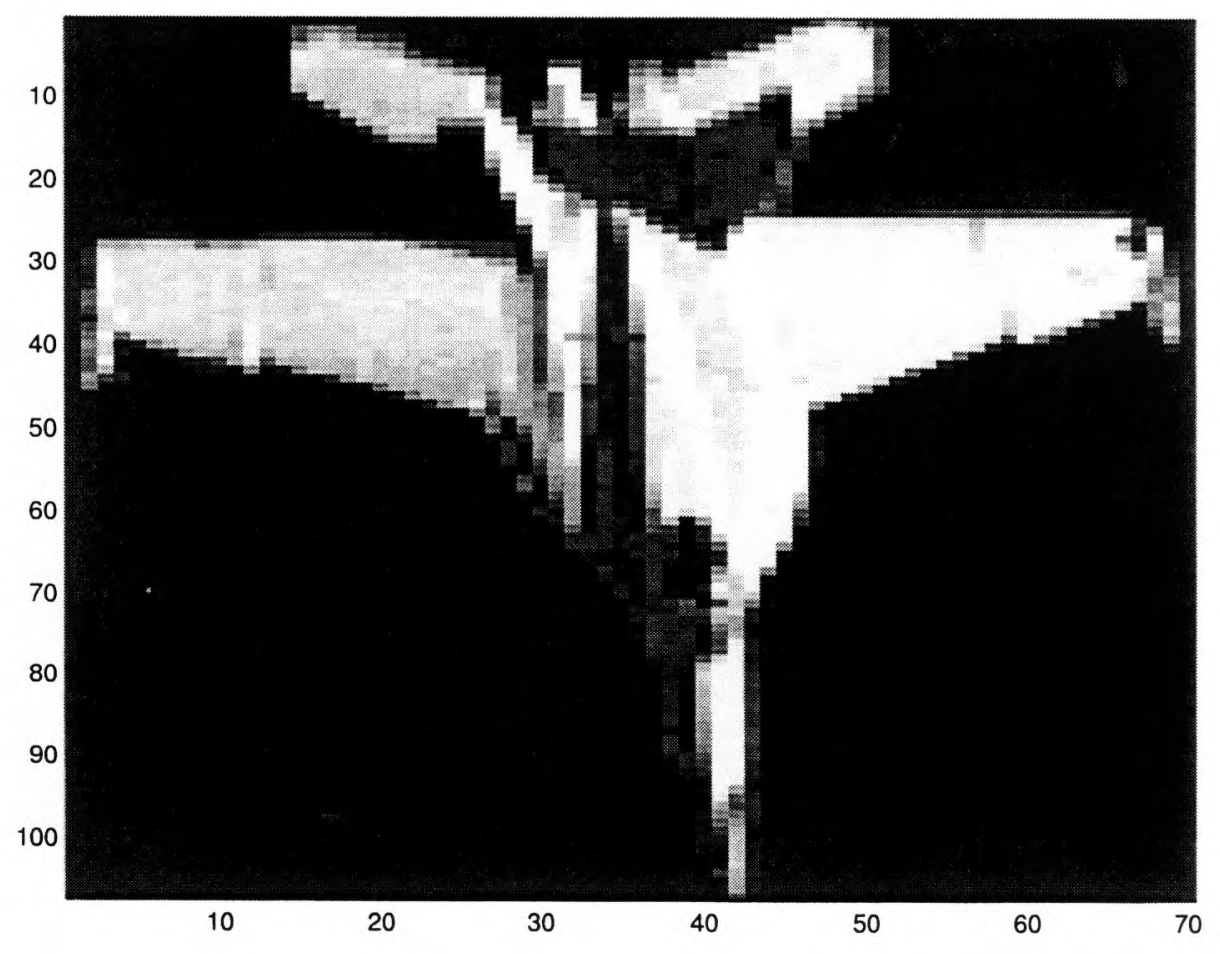

Figure 1: Target to be detected. 
256 / Critical Reviews Vol. CR74

Target with color background noise and AWGN $\mu=0, \sigma=0.5$

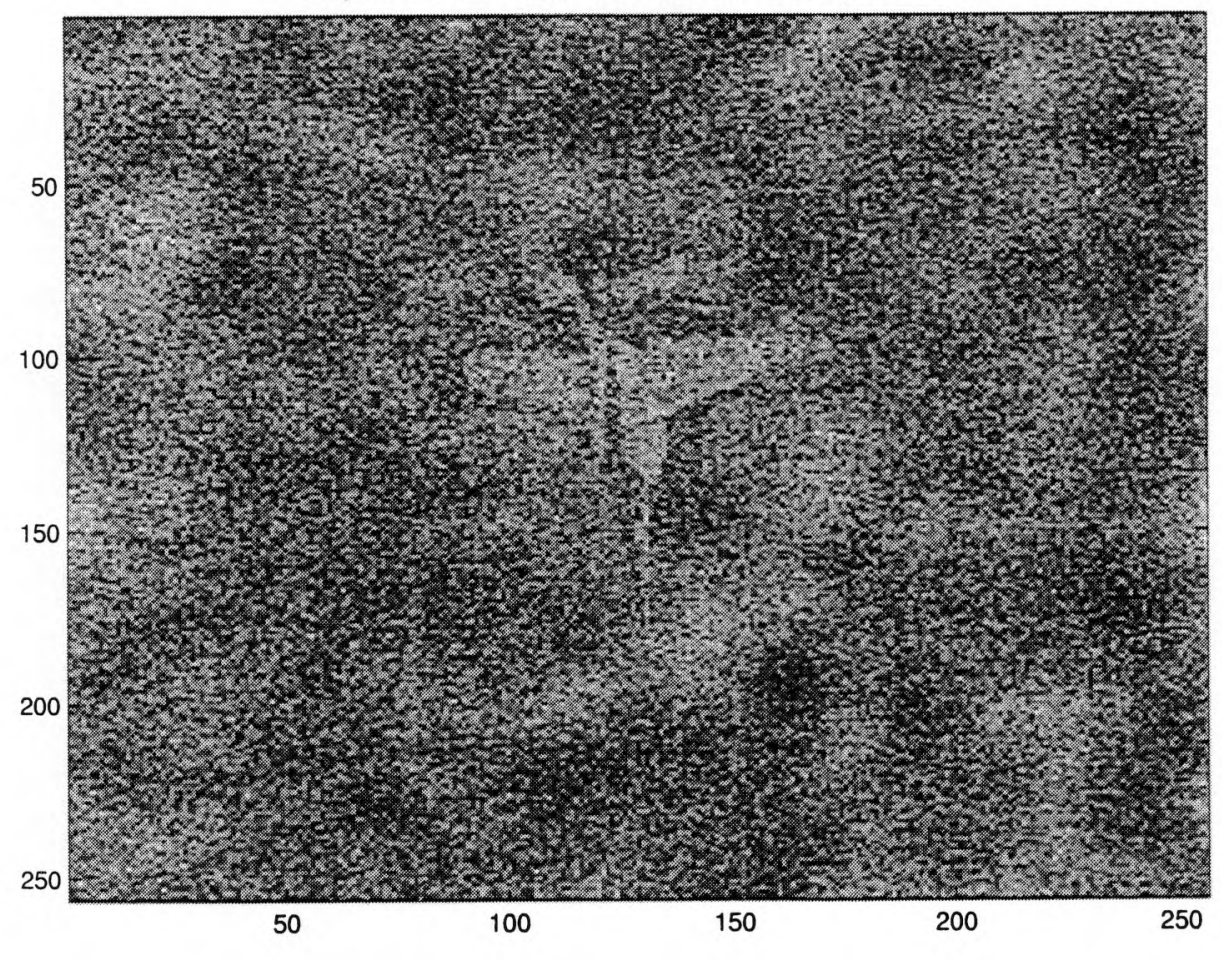

Figure 2: Input scene. 
1999 Euro-American Workshop on Optoelectronic Information Processing / 257

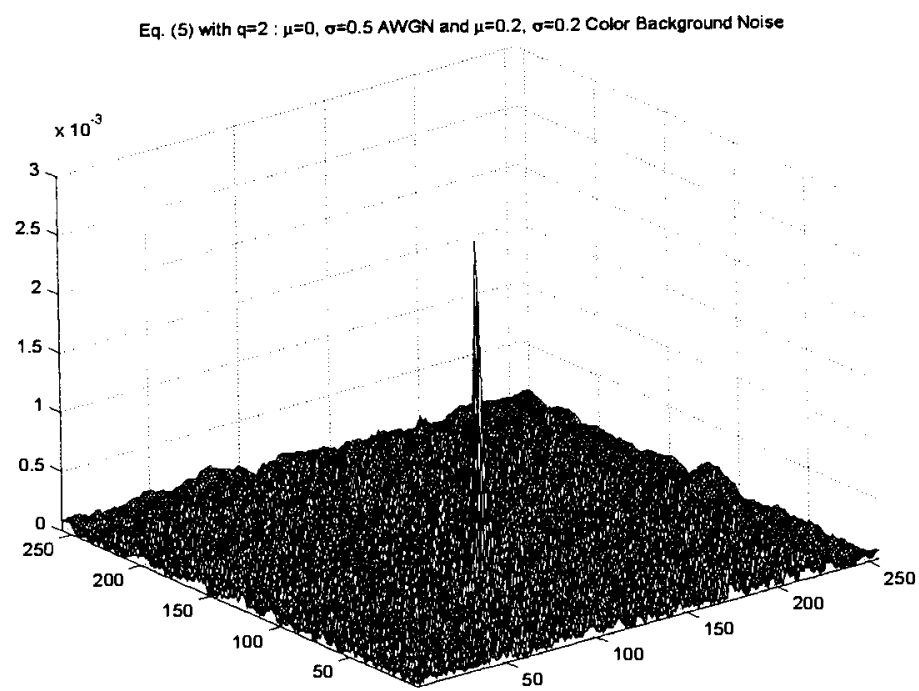

Figure 3: Output of the $l_{p}$-norm filter optimized for both discrimination capabilities and noise robustness (Eq. 5).

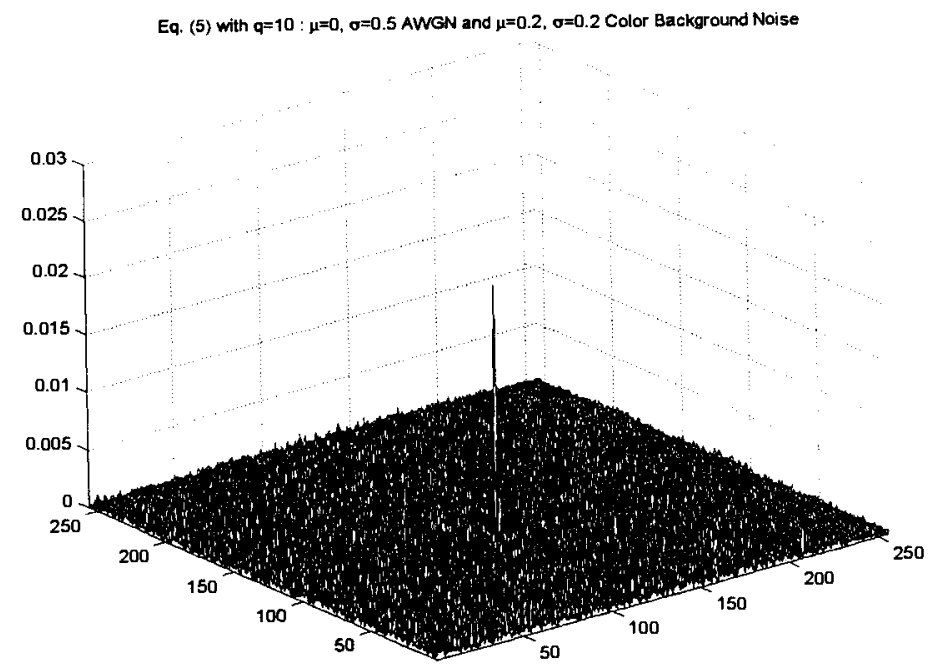

Figure 4: Output of the $l_{p}$-norm filter optimized for both discrimination capabilities and noise robustness (Eq. 5). 


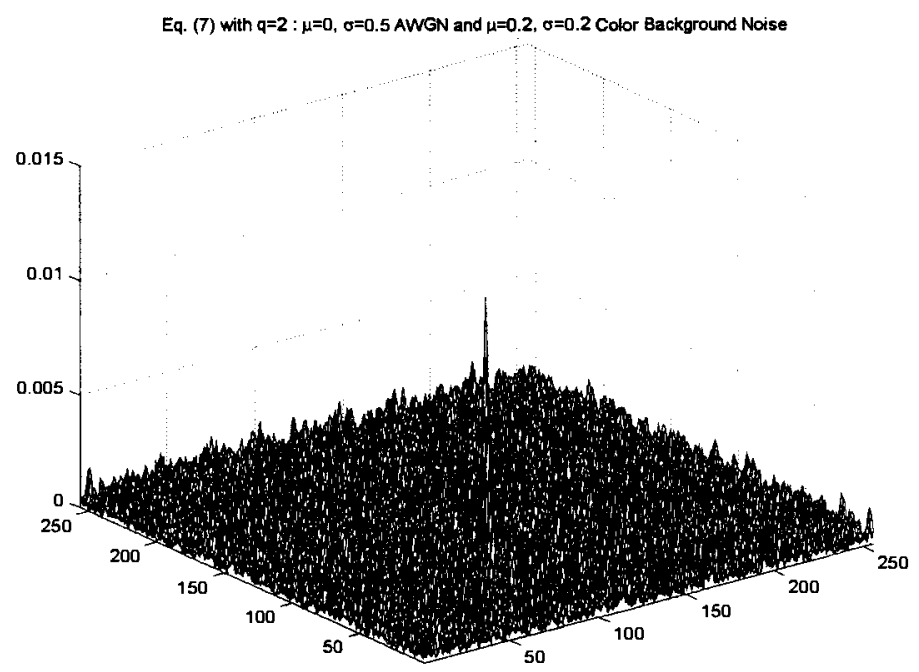

Figure 5: Output of the $l_{p}$-norm filter optimized for discrimination capabilities and noise robustness (Eq. 7).

norm of the scene input is minimized, we notice the higher values of peaks at non-target location.

Figures 8 through 10 show the output of the $H_{q}$ family of filters given by Eqs. (9) and (10). These filters are linear. Figure 8 is the output of the filter when $q=2$, figure 9 is the output of the filter when $q=10$, and figure 10 is the output of the filter when $q=\infty$, given by Eq. (10). The set of filters whose output is given by figures 8 through 10 were designed to optimize both the noise robustness and discrimination capabilities. Once again we see that the correlation peaks are sharper for larger values of $q$. Note that, when $q=2, H_{q}$ filter is the usual matched filter. 
1999 Euro-American Workshop on Optoelectronic Information Processing / 259

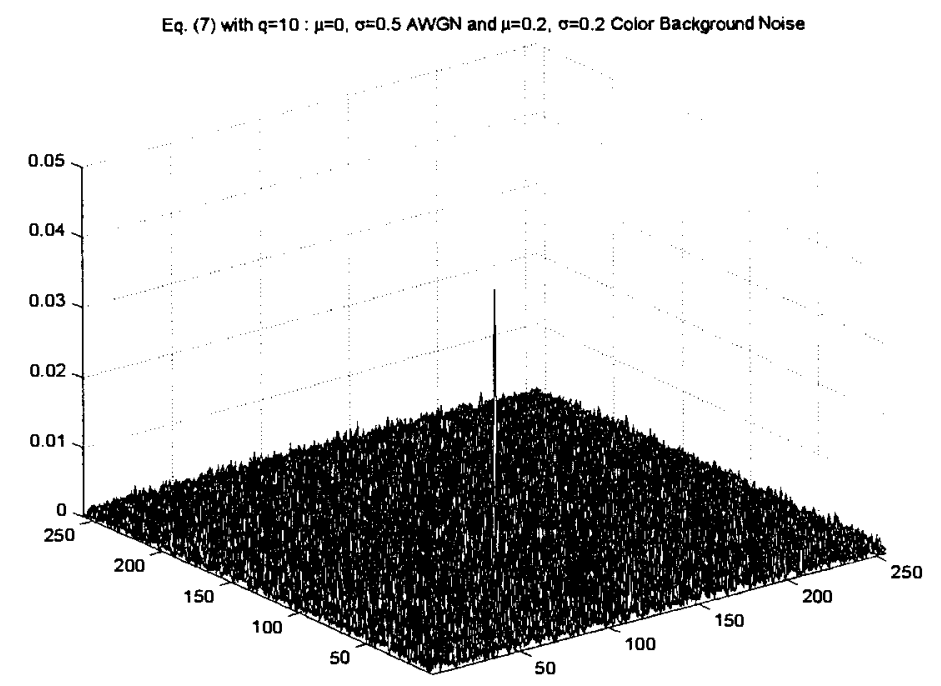

Figure 6: Output of the $l_{p}$-norm filter optimized for discrimination capabilities (Eq. 7).

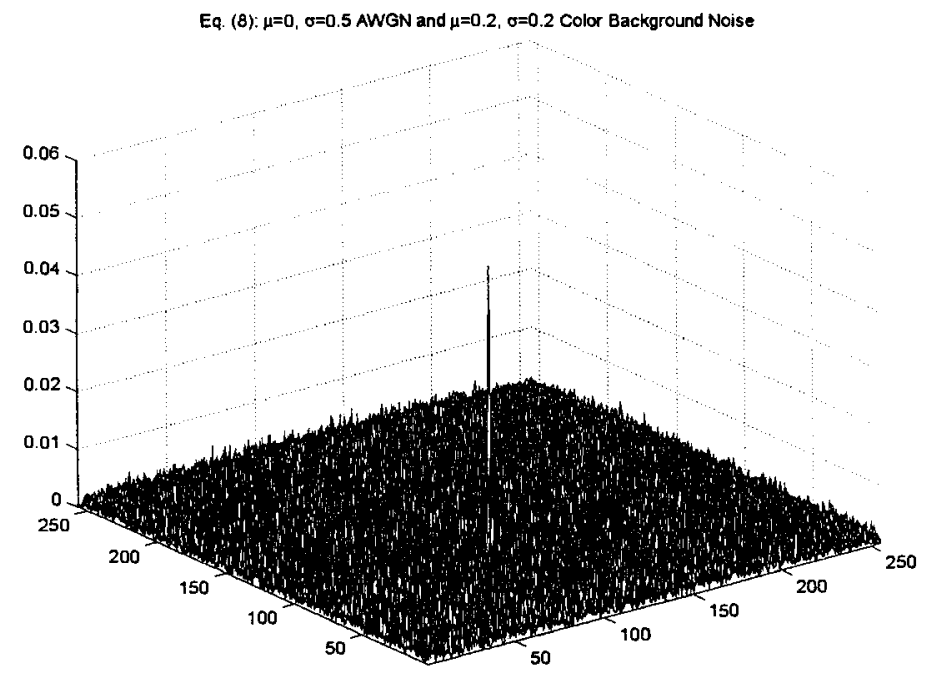

Figure 7: Output of the $l_{p}$-norm filter optimized for discrimination capabilities (Eq. 8). 
260 / Critical Reviews Vol. CR74

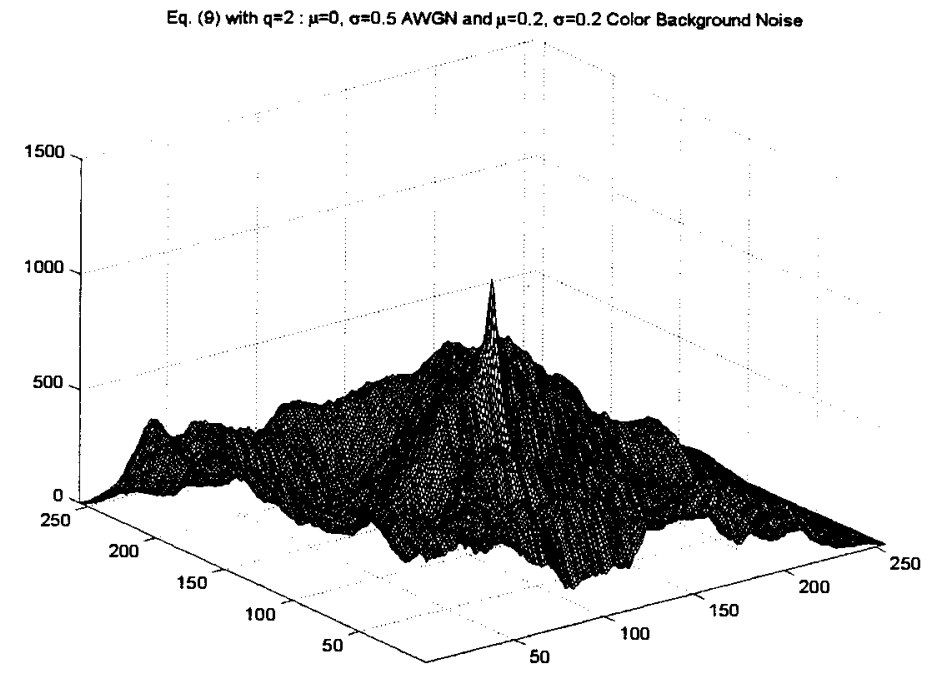

Figure 8: Output of the $l_{p}$-norm filter optimized for noise robustness (Eq. 9).

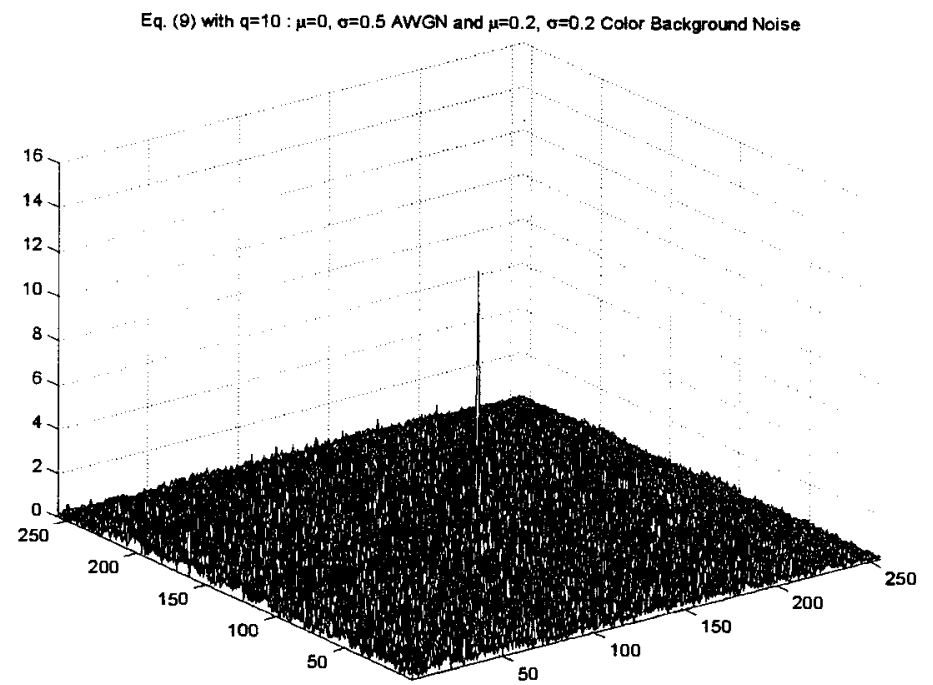

Figure 9: Output of the $l_{p}$-norm filter optimized for noise robustness (Eq. 9). 


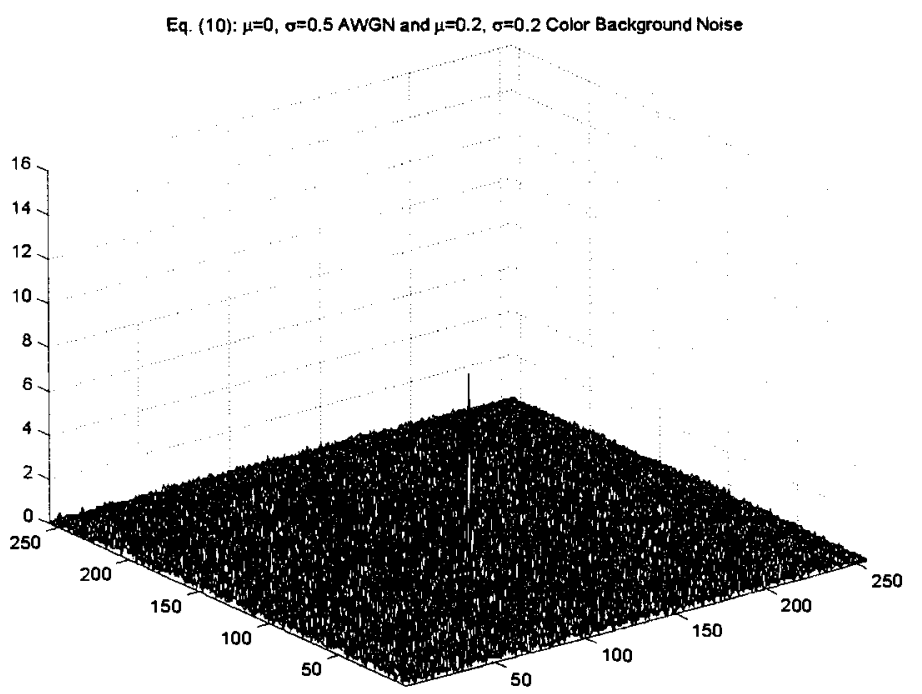

Figure 10: Output of the $l_{p}$-norm filter optimized noise robustness (Eq. 10).

\section{CONCLUSIONS}

We reviewed and extended the development of the $l_{p}$-norm optimum filters.

These filters were derived based on using an $l_{p}$-norm metric for arbitrary values of $p>1$, rather than the standard mean squared metric. $l_{p}$-norm criterion is used to derive filters to obtain greater freedom in adjusting noise robustness and discrimination capabilities.

These filters were obtained by minimizing the output due to noise and output to due the input signal, using $l_{p}$ norm as the metric, subject to certain constraint on the output of the filter when the input to the system is the target to be detected. The freedom in choosing the constraint on the output when the input to the system is the target to be detected allows the designer of the filter to emphasis or deemphasis certain range of the frequencies. 
The family of filters developed here gives a unified mathematical justification of the many of the well known, as well as, some of the more recently proposed filters.

We also tested the performance of the filters using computer simulation sand examined the discrimination capabilities of the filters for different values of $p$. The tests that we conducted show that, the filters performance (discrimination capabilities) improves when $p$ decreases. This is shown by sharp peaks at the target location.

\section{References}

[1] N. Towghi and B. Javidi, " $l_{p}$-norm optimum filters for image recognition," To appear in Journal of Opt. Soc. Am. A, Vol. 16, No. 8, 1999.

[2] J. L. Turin, "An Introduction to Matched Filters," IRE Trans. Inf. Theory, IT-6, 311-329, 1960.

[3] D. Casasent and D. Psaltis, "Position, rotation and scale invariant optical correlation," Appl Opt. 15, 1795-1799 1976.

[4] J. L. Horner and P. D. Gianino, "Phase-only matched filtering," Appl Opt. $\mathbf{2 3}, 812-816,1984$.

[5] H. J. Caufield and W. T. Maloney, "Improved discrimination in optical character recognition," Appl. Opt., 8, 2354, 1969. 
1999 Euro-American Workshop on Optoelectronic Information Processing / 263

[6] D. L. Flannery and J. L. Horner, "Fourier optical signal processor," Proc. of the IEEE 77, 1511, 1989.

[7] D. Casasent, "Unified synthetic discrimination function computational formulation," Appl. Opt. 23, 1620-1627 1984.

[8] Ph. Refregier and J. Figue, "Optimal trade-off filters for pattern recognition and their comparison with Wiener approach," Opt. Comput. process. 1 245-265, 1991.

[9] K. H. Fielding and J. L. Horner, "1-f binary joint transform correlator," Opt. Eng. 29, 1081-1087, 1990.

[10] B. Javidi, "Nonlinear joint power spectrum based optical correlation," Appl. Opt.28, 2358-2367, 1989.

[11] Ph. Refregier, V. Laude, and B. Javidi, "Nonlinear joint-transform correlation: an optimal solution for adaptive image discrimination and input noise robustness," Opt. Lett. 19(6), 405-407, 1994.

[12] B. V. K. Vijaya Kumar, "Tutorial survey of composite filter designs for optical correlators," Appl. Opt. 31 4773-4800, 1992.

[13] A. Mahalanobis, B. V. K. Vijaya Kumar, and D. Casasent, "Minimum average correlation energy filters," Appl. Opt. 26 3633-3640, 1987.

[14] $\mathrm{Ph}$. Refregier, "Filter design for optical pattern recognition: multicriteria approach," Opt. Lett. 15, 854-856, 1990. 
264 / Critical Reviews Vol. CR74

[15] Ph. Refregier, "Optimal trade-off filters for noise robustness, sharpness of the correlation peak, and Horner efficiency," Opt. Lett. 16, 829-831, 1991.

[16] Ph. Refregier, "Optical pattern recognition: Optimal trade-off circular harmonic filters," Opt. Commun. 86, 113-118, 1991.

[17] M. S. Millan, E. Perez, and K. Chalasinska-Macukow, "Pattern recognition with variable discrimination capability by dual nonlinear optical correlation," Opt. Commun., 161 115-122, 1999. 